\title{
The DAC system and associations with multiple myeloma
}

\author{
Enrique M. Ocio • Jesús F. San Miguel
}

Received: 27 October 2010 / Accepted: 28 October 2010 / Published online: 1 December 2010

(C) The Author(s) 2010. This article is published with open access at Springerlink.com

Summary Despite the clear progress achieved in recent years in the treatment of MM, most patients eventually relapse and therefore novel therapeutic options are still necessary for these patients. In this regard, several drugs that target specific mechanisms of the tumor cells are currently being explored in the preclinical and clinical setting. This manuscripts offers a review of the rationale and current status of the antimyeloma activity of one of the most relevant examples of these targeted drugs: deacetylase inhibitors (DACi). Several studies have demonstrated the prooncogenic activity of deacetylases (DACs) through the targeting not only of histones but also of non histone proteins relevant to tumor progression, such as p53, E2F family members, Bcl-6, Hsp90, HIF-1 $\alpha$ or Nur77. This fact together with the DACs overexpression present in several tumors, has prompted the development of some DACi with potential antitumor effect. This situation is also evident in the case of MM as two mechanisms of DACi, the inhibition of the epigenetic inactivation of $\mathrm{p} 53$ and the blockade of the unfolded protein response, through the inhibition of the aggressome formation (by targeting DAC6) and the inactivation of the chaperone system (by acetylating HSP90), provides the rationale for the exploration of the potential antimyeloma activity of these compounds. Several DACi with different chemical structure and different

E. M. Ocio · J. F. San Miguel $(\bowtie)$

University Hospital of Salamanca,

Salamanca, Spain

e-mail: sanmigiz@usal.es

E. M. Ocio - J. F. San Miguel

IBMCC-CSIC (Institute of Molecular and Cellular Biology

of Cancer-Spanish National Research Council) Cancer Research

Center, University of Salamanca,

Salamanca, Spain selectivity for targeting the DAC families have been tested in MM. Their preclinical activity in monotherapy has been quite exciting and has been described to be mediated by various mechanisms: the induction of apoptosis and cell cycle arrest mainly by the upregulation of $\mathrm{p} 21$; the interferece with the interaction between plasma cells and the microenvironment, by reducing the expression and signalling of several cytokines or by inhibiting angiogenesis. Finally they also have a role in protecting murine models from myeloma bone disease. Neverteless, the clinical activity in monotherapy of these drugs in relapsed/refractory MM patients has been very modest. This has prompted the development of combinations such as the one with bortezomib or lenalidomide and dexamethasone, which have already been taken into the clinics with positive preliminary results.

Keywords DACi $\cdot$ HDACi $\cdot$ Deacetylases $\cdot$ Multiple myeloma $\cdot$ Bortezomib $\cdot$ Lenalidomide $\cdot$ Dexamethasone

\section{Introduction}

Multiple Myeloma (MM) is a B cell malignancy characterized by the presence of bone marrow infiltration by clonal plasma cells that generally secrete a monoclonal component in the serum or urine [1]. It is the second most frequent haematological malignancy, after non hodgkin lymphomas, and accounts approximately for a $10 \%$ of all haematological tumors and $1 \%$ of all cancers [2]. Treatment of MM has remained substantially unchanged for some time (reviewed in [3]) with alkylating agents such as melphalan in combination with steroids being the gold standard for more than 25 years. Later on, in the 1970 decade, other drugs such as carmustine or vincristine where combined with melphalan, cyclophosphamide or steroids giving rise to the 
polichemotherapeutic regimens; and 10 years later high doses of melphalan with autologous stem cell transplantation (ASCT) where introduced into the clinical practice. All these treatment schemes resulted in an overall survival of around 30 months until 1994 with a slight improvement in the subsequent 5 years, probably due to the introduction of ASCT and better measures of supportive care [4]. Finally, in the first decade of this century, some new drugs with novel mechanisms of action and clear antimyeloma activity have been discovered and approved. In this regard, several studies have demonstrated the activity of the proteasome inhibitor bortezomib [5] and the immunomodulatory agents thalidomide [6] and lenalidomide [7, 8] in relapsed/ refractory MM patients. The emergence of these drugs has resulted in a clear improvement in the outcome of these patients in the last years, with an increase in median overall survival of up to 5 years [4].

Nevertheless, despite this clear progress, most patients (if not all) eventually relapse and therefore novel therapeutic options are still necessary for these relapsed or refractory patients. In this regard, several drugs that target specific mechanisms of the tumoral cells are currently being explored in the preclinical and clinical setting $[9,10]$. Some examples of these targeted agents are secondgeneration proteasome inhibitors or immunomodulatory agents, inhibitors of the PI3K/AKT/mTOR pathway, heat shock protein inhibitors, several monoclonal antibodies against surface antigens such as CS1 and deacetylase inhibitors (DACi), the focus of this review.

\section{Concept and classification of DACs and DACi}

Deacetylases (DACs) are enzymes specialized in removing acetyl groups from their target proteins. As histones were formerly considered the main client proteins for DACs, these enzymes are sometimes known as histone deacetylases (HDACs) [11, 12] but we currently know that DACs have a dual role; on one side, they have the epigenetic regulatory function, which is exerted by controlling the delicate balance between acetylation and deacetylation of histones that control the accessibility of the chromatin to transcription factors [11]. On the other hand, many other non-histone proteins such as $\alpha$-tubulin, p53, p73, retinoblastome, several steroid receptors, E2F family members, Bcl-6, Hsp90, HIF-1 $\alpha$ or Nur77 among others, are also deacetylated by DACs; in fact, more that 50 non-histone proteins have been described to be substrates of DACs [12].

Based on their homology with yeast proteins DACs have been typically classified into four groups: $[13,14]$ class I, II and IV DACs are called classical DACs, whereas class III DACs are called sirtuins due to their of homology to yeast
Sir2, and display characteristic features, such as the requirement of $\mathrm{NAD}^{+}$as an essential cofactor for their activity, and the absence of Zinc in the catalytic site [11, 13]. The classical DACs are the ones that have been implicated in oncogenesis and are targets of the DACi currently used in the clinic so we will mainly focus in these three types of DACs. Class I DACs includes DAC1, DAC2, DAC3 and DAC8. They are commonly restricted to the nucleus of the cells [15-17] and their most relevant substrates are proteins such as p53, MEF2D, Stat3 or E2F. Class II DACs can be further subdivided into class IIA and IIB, which display different targets and localization. Class IIA are both nuclear and cytoplasmic DACs and include DAC4, DAC5, DAC7 and DAC9. The class IIB are probably more important and contains the cytoplasmic DAC6 and DAC10. The first one has been described to be very important in tumour pathogenesis and specifically in MM mainly due to their activity on two relevant substrates, $\alpha$-tubulin $[18,19]$ and Hsp-90 [20]. Therefore, as we will review later in the manuscript, the inhibition of this DAC may have a significant role in the treatment of MM. The last type of classic DAC are class IV DACs, whose only representative is DAC11 whose function and role in oncogenesis has not been well described yet.

Several factors have prompted the use of DACi in the treatment of cancer: First, the increasing importance given to epigenetic modifications in the pathogenesis of cancer [21] and the role that changes in the activity of the some non-histone substrates of DACi also play in oncogenesis. Moreover, in support of this, significant changes in the expression of DACs have been described in different tumors. For instance, class I DACs, principally DAC 1, 2 and 3 , are overexpressed in gastrointestinal and prostatic tumors [22-25] and this is associated with an adverse prognosis. Class IIA DACs expression has been shown to be also upregulated in different tumors: DAC 4 in breast and DAC 5 and DAC 7 in colorectal cancer [26]. Regarding DAC6 it is significantly overexpressed and is associated with advance stage in oral squamous cell carcinoma [27]. By contrast, the upregulation of its expression in breast cancer has been described to confer a better prognosis [28, 29]. A second important reason that specifically supports the use of HDACi in haematological malignancies is that functional changes in these DACs have been described in several leukemias and lymphomas. In this regard, DACs are frequently involved in some oncogenic translocations present in these diseases; the AML-1-ETO translocation, characteristic of M2 acute myeloid leukemia modulates repressive cofactors such as histone deacetylases (HDACs) and DNA methyltransferases (DNMTs) [12, 30-32]. Both $\mathrm{DAC} 3$ and DAC4 are recruited by the PML/RAR $\alpha$ translocation in acute promyelocytic leukemia, and collaborate in the repression of transcription that provokes the 
loss of differentiation characteristic of this type of leukemia $[33,34]$.

Regarding myeloma the activity of DACs on several non-histone proteins provides a good rationale for the use of these compounds in this disease. This is the case of p53 that is considered a very important tumor suppressor gene for all malignancies, but specifically for multiple myeloma. In fact TP53 deletion, that hampers the transcription of functional protein [35], is one of the main adverse prognostic factors in this disease [36-38]. Moreover, the antimyeloma activity of some drugs has been, at least in part related to the increase in the protein levels of p53 [39]. This data together with the fact that the reversal of the epigenetic inactivation of this protein has also been demonstrated to induce apoptosis [40] points out p53 as a potential relevant antitumoral target mechanism for DACi in MM. Another pathogenetic mechanism in MM is the unfolded protein response. This mechanism allows the correct management of the great amount of proteins synthesized by tumor cells. This situation is critical in $\mathrm{MM}$ as this disease is defined by the proliferation of plasma cells, which are characterized by the secretion of high quantities of a protein, the monoclonal immunoglobulin. For this purpose the cell requires the correct functionality of the molecular chaperone HSP-90 (Heat Shock Protein-90) that allows the correct folding of the synthesized proteins into their spatial conformation [41, 42]. When this mechanism is overloaded, there is an increase in misfolded proteins that have to be degraded by the proteasome through their previous ubiquitination. Nevertheless, those misfolded proteins, which cannot be degraded by the proteasome form stable aggregates that are toxic to the cell. An alternative system to the proteasome for degradation of poliubiquitinated misfolded proteins, termed the aggresome has been recently described [43, 44]. DAC6 facilitates the interaction between this aggregates and the dynein motors in order to form the aggresomes [45] that are structures that eliminate this toxic proteins from the cell. As we have seen before, DACs have an important role in this unfolded protein response at least through two different pathways: the deacetylation of HSP-90 that allows its correct functioning and the formation of aggresomes by DAC6, and therefore its inhibition with DACi could have a role in MM treatment.

All these data has stimulated the investigation of DAC inhibitors as potential anticancer drugs and several of these molecules are already at different stages of preclinical and clinical development. Nevertheless, not all these DACi under development have the same properties, in fact they can be divided into different groups according to their chemical structure and their pattern of DAC inhibition [12, $14,15,46,47]$. The first group of agents to be used as DACi are aliphatic acids. It includes agents that have previously been used in the clinic for other indications based on other mechanisms of activity. The most important ones are valproic acid, phenylbutyrate or butyrate. They mainly inhibit class I DACs but may have some effect on class II A HDACs. Neverteless, their potency of inhibition of DAC is quite low and therefore their clinical activity has not been very important. Two classes of DACi are quite specific of the nuclear class I DACs with very scarce or no effect over the cytoplasmic DACs. These are cyclic peptides, with romidepsin (FK-228) being the most significant representative of this group. The second group is constituted by benzamides such as entinostat (MS-275) or MGCD0103. Hydroxamates are derivatives of the hydroxamic acid and are characteristically pan-DACi because they inhibit both class I and II HDACs. Due to its potency and breadth of activity and the potency of its components, this is the group with a more extensive development. It includes molecules such as vorinostat (SAHA), panobinostat (LBH589), TSA (Trichostatin A), belinostat (PDX-101), resminostat (RAS2410), LAQ824 or ITF2357. A different small molecule that does not fit into any of the previous classes is tubacin, which was recently described as an specific inhibitor of DAC6 [48] and induces acetylation of tubulin and Hsp-90, without affecting histone acetylation.

\section{Antimyeloma activity of DACi in monotherapy}

Preclinical data and mechanisms of action of DACi in MM

Based on the previous data, several studies have explored the in vitro and in vivo activity of different DACi as single agents in MM. This is the case of vorinostat [49, 50], sodium butyrate and trichostatin A [51], LAQ824 [52], depsipeptide [53], valproic acid [54-57], panobinostat [5860], belinostat [61], R306465 [62], KD5170 [63], JNJ26481585 [64], ITF2357 [65] or resminostat [66]. Regarding the mechanism of action of DACi in MM, these compounds act through the induction of apoptosis mainly by a caspase dependent mechanism but also a caspase independent apoptotic mechanism has been described for some of them $[49,56,59]$. Interestingly, a cell cycle arrest in $\mathrm{G} 0 / \mathrm{G} 1$ phases has been demonstrated as a very common effect of most DACi analyzed. This effect seems to be, at least partially mediated through the upregulation of the tumor suppressor gen p21, mechanism that has been observed with most of the DACi analyzed independently of the type and pattern of DAC inhibition [49, 51, 57, 59, $62,64,66,67]$. This p21 deregulation has also been associated with a decrease in the levels of some proliferative proteins such as cyclin $\mathrm{D}[54,66]$, Cdk4 [66] or pRb $[49,51,66]$. The homogeneity observed with these drugs in the cell cycle regulation through p21 modifications 
suggests that it may be a class specific effect of DACi in MM cells, and probably all tumor cells. A parallel mechanism which may be related to the previous one is the interference with the pAKT pathway that has been demonstrated by the decreased phosphorylation of 4E-BP1 and $\mathrm{p} 70 \mathrm{~S} 6 \mathrm{k}$, and that has been recently described with resminostat [66]. This blockade of the AKT pathway also leads to a reduction in proliferation of tumor plasma cells. Two DACi with different chemical structure, belinostat and KD5170 have also been shown to induce DNA damage as assessed by $\mathrm{H} 2 \mathrm{AX}$ phosphorylation [61, 63]. This effect was preceded by the induction of oxidative stress with generation of reactive oxygen species and a subsequent release of mitochondrial mediators of apoptosis.

As important as the discovery of the mechanisms of action of a drug or type of drugs is the detection of potential mechanisms of resistance for these compounds. A second mechanism for DACi, which has mainly been observed in hydroxamate-derived compounds, is the modulation of several bcl-2 family members leading to a proapoptotic phenotype. In this regard, several studies have demonstrated that treatment with these drugs induces a downregulation of several antiapoptotic member of this family of proteins, mainly Bcl-2 and Bcl-X. This has been the case of vorinostat [49, 67, 68], panobinostat [59], depsipeptide [53] or trichostatin [67]. Moreover, treatment with vorinostat has been associated with an increase in the expression of some proapoptotic $\mathrm{BH} 3$-only proteins such as Bim, Bak, Bax, Puma and Noxa [67, 69]. Interestingly, the overexpression of some of these antiapoptotic proteins was able to prevent DACi induced cell death. In this regard, the overexpression of Bcl-X in MM cells inhibited panobinostat induced apoptosis [59], and transfection of Bcl-2 cDNA into MM cells completely abrogated the activity of vorinostat [49]. These observations clearly indicate the relevant importance of the modulation of Bcl-2 family members in the activity of DACi and point out to a possible mechanism of resistance of the cells to these treatments.

A very important player in MM pathogenesis is the interaction of the tumor cells with the microenvironment. In fact this interaction through direct contact or through the secretion of cytokines triggers the activation of different signaling pathways such as the PI3K/AKT, MAPK/MEK/ ERK or JAK/STAT pathways, that ends up in a proliferative advantage for myelomatous plasma cells and a drug resistance phenotype [70, 71]. As a consequence, in the last years, there has been an increasing interest in developing novel treatments that could act not only on the tumor cell, but also on the microenvironment and on the interactions between these two factors. Several preclinical results suggest that DACi have a role in blocking this mechanism in different ways. First, panobinostat and LAQ824 are able to kill MM cells even when they are cocultured with stromal cells $[52,58]$. Regarding the effect of DACi on cytokines, ITF2357 downregulated the receptor alfa of IL-6 leading by a decreased signaling as measured by STAT-3 phosphorylation in the presence or absence of IL-6 [65]. Moreover, GEP analysis of changes induced after vorinostat treatment indicated that this drug downregulated transcripts for members of the Insulin-like growth factor/ insulin-like growth factor-1 receptor (IGF/IGF-1-R) and IL6 receptor [50], and functional experiments showed that this same drug inhibited the secretion of IL-6 induced in by the bone marrow stromal cells after binding to MM cells [49].

Two other factors to be taken into account when analyzing the role of the microenvironment in $\mathrm{MM}$ are angiogenesis, and MM bone disease, which is one of the most debilitating symptoms for these patients and results from the disruption of the delicate equilibrium between formation of bone by osteoblasts and the bone resorption induced by osteoclasts. Regarding these two aspects, treatment of 5T2MM-bearing mice with JNJ-26481585 resulted in a significant decrease in angiogenesis and a significant reduction in the development of bone disease [64]. In the same line, panobinostat was able to decrease trabecular bone density loss in a model of disseminated myeloma [60]. Valproic acid also has antiangiogenic effect as assessed by the inhibition of the production of vascular endothelial growth factor [54], and the inhibition of vascular tubule formation enhanced by the co-culture of MM cells with osteoclasts [56]. Finally, treatment with this drug also suppressed osteoclastogenesis and osteoclastmediated MM cell growth [56].

\section{Clinical}

Four studies have analyzed the activity of DACi as single agents in phase I/II trials in relapsed/refractory MM (Table 1). All of them included small numbers of patients which were quite refractory to their previous therapies. The
Table 1 Summary of the most relevant results of clinical trials with DACi in monotherapy in MM

\begin{tabular}{llllll}
\hline DACi & Reference & Phase & $\mathrm{n}$ & ORR & Responses \\
\hline Panobinostat & Wolf 2008 [72] & II & 38 & $3 \%$ & 1PR, 1 MR, 1 SD \\
Vorinostat & Richardson 2008 [73] & I & 13 & $10 \%$ & 1 PR, 9 SD \\
ITF2357 & Gally 2010 [74] & II & 19 & $7 \%$ & 1 PR, 1 SD \\
Romidepsin & Niesvizky 2005 [75] & II & 12 & $0 \%$ & 11 SD \\
\hline
\end{tabular}


results show that, despite their preclinical activity, the clinical efficacy of all these drugs, when used in monotherapy, was quite modest.

Panobinostat monotherapy was explored in a phase II trial in 38 patients who had received at least 2 prior lines of therapy (including bortezomib and lenalidomide or thalidomide) and who were also refractory to their most recent line of therapy. Among them one patient achieved a durable VGPR and another one a durable MR; in addition three patients achieved disease stabilization. Overall, panobinostat was well tolerated, with half of the patients suffering from GI symptoms as well as fatigue/asthenia and cytopenias [72]. In a phase I trial of escalating doses of oral vorinostat, 13 patients were enrolled with one of them achieving minimal response and nine stable disease. The most frequent drug-related adverse events were mostly mild and included general symptoms (fatigue, anorexia) and GI symptoms (dehydration, diarrhea, and nausea) [73]. A phase-II trial of the oral DACi ITF2357 was performed in 19 patients with relapsed or progressive MM with oral dexamethasone allowed to a maximum weekly amount of $20 \mathrm{mg}$. No patients responded with 5 of them showing stable disease. The most frequent toxicities were GI, transient electrocardiographic abnormalities and thrombocytopenia [74]. Finally, the cyclic peptide romidepsin has also been explored in monotherapy in a phase II trial in MM patients with documented progressive disease. Twelve patients with relapsed or refractory MM were treated. Eleven out of the 12 patients had stable disease (SD) with similar toxicity to that reported with the previous DACi [75].

\section{Combinations of DACi}

In spite of the rather disappointing results obtained with DACi used as single agents in the relapse/refractory setting, the observation of disease stabilization and some responses, together with the in vitro data showing marked synergism in combination with other antimyeloma agents, set up the basis for the investigation of the role of DACi in combination therapies (Table 2).

The rationale for the combination of DACi with proteasome inhibitors relies in a mechanism that has been previously described in this review: the simultaneous targeting of several proteins involved in the unfolded protein response. By blocking the proteasome with specific inhibitors we block the degradation of the ubiquitinated misfolded proteins by this organelle, while the use of DACi interfere with the activity of heat shock proteins, necessary for the correct folding of proteins, and with the aggressome formation (through the inhibition of DAC6) which is also important in the elimination of toxic misfolded proteins. Therefore, the simultaneous blockade of this three survival mechanisms would end up in a synergistic cytotoxic effect. In this regard, several studies have demonstrated the potentiation of the activity of DACi with bortezomib [58, 60, $61,63,66,76-79]$ and this potentiation was even stronger when associating dexamethasone [60]. Interestingly, a significant deregulation of genes involved in cell cycle arrest, apoptosis and other pathways such as chemotaxis or adhesion were observed after treatment with the triple combination with bortezomib and dexamethasone when compared to these agents in monotherapy [60]. Two phase I trials with vorinostat in combination with bortezomib have been reported [80, 81] including 23 and 34 patients, with an ORR of around $40 \%$. Interestingly, the combination was able to induce responses in patients that had relapsed or had been previously refractory to bortezomib. In this regard, in the first study three PR were observed in 9 bortezomib refractory patients [80], and in the second one, among 13 patients previously exposed to bortezomib 5 achieved PR, 1 MR and 7 SD [82]. Panobinostat has also been combined with bortezomib in this relapsed/ refractory setting in a phase $\mathrm{Ib}$ trial that included 29 patients. The ORR was $48 \%$ [83] with some patients achieving CR. This combination was also effective in 10 bortezomib refractory patients with $4 \mathrm{PR}$ and $2 \mathrm{MR}$. The third DACi that has been combined with bortezomib is the cyclic peptide, romidepsin [84]. Twenty five patients were included in this

Table 2 Summary of the most relevant results of clinical trials with DACi in combination with other agents in MM

\begin{tabular}{lllllll}
\hline DACi & Combination & Reference & Phase & $\mathrm{n}$ & ORR & Responses \\
\hline Vorinostat & Bortezomib & Badros 2009 [80] & $\mathrm{I}$ & 23 & $42 \%$ & \\
Vorinostat & Bortezomib & Weber 2008 [81] & $\mathrm{I}$ & 34 & $43 \%$ & $10 \% \mathrm{VGPR}, 33 \% \mathrm{PR}, 48 \% \mathrm{SD}$ \\
Vorinostat & Bortezomib & Weber 2008 [82] & $\mathrm{I}$ & $13^{\mathrm{a}}$ & $38 \%$ & $38 \% \mathrm{PR}, 8 \% \mathrm{MR}, 54 \% \mathrm{SD}$ \\
Panobinostat & Bortezomib & San Miguel 2009 [83] & $\mathrm{Ib}$ & 29 & $48 \%$ & $14 \% \mathrm{CR}, 34 \% \mathrm{PR}, 14 \% \mathrm{MR}$ \\
Romidepsin & Bortezomib & Harrison 2008 [84] & $\mathrm{I}$ & 25 & $67 \%$ & $22 \% \mathrm{CR}, 22 \% \mathrm{VGPR}, 22 \% \mathrm{PR}, 28 \% \mathrm{MR}$ \\
Vorinostat & Pegylated Lip. Dox + Bortezomib & Voorhees 2009 [85] & $\mathrm{I}$ & 11 & $63 \%$ & $9 \% \mathrm{CR}, 27 \% \mathrm{VGPR}, 27 \% \mathrm{PR}$ \\
Panobinostat & Lenalidomide + Dex & Spencer 2009 [86] & $\mathrm{Ib}$ & 13 & $45 \%$ & $8 \% \mathrm{SCR}, 8 \% \mathrm{CR}, 30 \% \mathrm{VGPR}$ \\
Vorinostat & Lenalidomide + Dex & Siegel 2009 [87] & $\mathrm{I}$ & 28 & $46 \%$ & $7 \% \mathrm{CR}, 39 \% \mathrm{PR}, 18 \% \mathrm{MR}, 21 \% \mathrm{SD}$ \\
Panobinostat & Melphalan & Berenson 2009 [88] & $\mathrm{I}$ & 15 & $27 \%$ & $7 \% \mathrm{CR}, 20 \% \mathrm{VGPR}$ \\
\hline
\end{tabular}

${ }^{\mathrm{a}}$ All patients were previously exposed to Bortezomib 
study and an ORR of $67 \%$ was observed. Two-thirds of these responses were of good quality (CR or VGPR), figure that is important in this setting of relapsed/refractory patients. Regarding toxicity, the combination of DACi and bortezomib was overall well tolerated, with general and GI symptoms probably derived from the use of the $\mathrm{DACi}$, and with reversible thrombocytopenia as the main specific toxicity of the combination. It is interesting to remark the promising activity on patients previously exposed to bortezomib, which highlights the synergy of the combination and the possibility to overcome bortezomib refractoriness. A further step was to add pegylated liposomal doxorubicine to this combination. Although numbers are very small, a clinical trial using this triple combination in 11 relapsed/refractory patients, most of them previously exposed to bortezomib and anthracyclins, showed $63 \%$ ORR with more than one-third of patients achieving VGPR or better [85].

A second interesting combination is the one with the immunomodulatory drug lenalidomide and dexamethasone. There are some in vitro results that support this association with combination indexes in the very high synergistic range [60]. Similarly to what has been said for the combination with bortezomib and dexamethasone, there is a transcriptomic rationale behind the synergy of this combination with a completely different array of genes deregulated by the triplet as compared to sum of the genes deregulated by the agents in monotherapy [60]. Both panobinostat and vorinostat have been combined with lenalidomide and dexamethasone in two phase I clinical trials [86, 87]. Both trials showed an ORR of around $45 \%$ with some patients achieving good quality responses: $7 \% \mathrm{CR}$ in the combination with vorinostat and $46 \%$ of VGPR or better for the combination that included panobinostat. Moreover, in the trial with vorinostat, among 12 patients that had received previous lenalidomide, four patients achieved PR or MR and $3 \mathrm{SD}$. The most frequent adverse effects for these combinations included general symptoms and GI symptoms such as diarrhoea, and also myelosuppression with different cytopenias (thrombocytopenia and neutropenia).

One last combination that has recently been tested is the one of panobinostat and melphalan. This combination showed onethird of responses in a phase I trial that enrolled 15 relapsed/ refractory MM patients, the majority of whom had previously been exposed to melphalan. In fact, all responses were observed in melphalan treated patients [88].

\section{Conclusions}

The solid preclinical rationale for the use of DACi in MM as well as the good positive clinical results, particularly in combination with other agents such as bortezomib or lenalidomide, suggest that this class of drugs may reach approval as antimyeloma agents. In this time, phase III randomized trials are underway in order to prove if the combination of DACi with either bortezomib or lenalidomide plus dexamethasone are superior to the standards used at present for relapsed/refractory patients.

Conflicts of interest Dr. Enrique Ocio has participated in advisory boards for Novartis Oncology and Celgene.

Dr. Jesús F. San Miguel has participated in advisory boards from Novartis, MSD, Celgene, and Janssen-Cilag, and has received research funding from Novartis Oncology.

Logistical support during the submission process of this article was provided by Springer Healthcare LLC. This support was funded by Novartis.

Open Access This article is distributed under the terms of the Creative Commons Attribution Noncommercial License which permits any noncommercial use, distribution, and reproduction in any medium, provided the original author(s) and source are credited.

\section{References}

1. Kyle RA, Rajkumar SV (2004) Multiple myeloma. N Engl J Med 351:1860-1873

2. Petrelli NJ, Winer EP, Brahmer J et al (2009) Clinical Cancer Advances 2009: major research advances in cancer treatment, prevention, and screening-a report from the American Society of Clinical Oncology. J Clin Oncol 27:6052-6069

3. Kyle RA, Rajkumar SV (2008) Multiple myeloma. Blood 111:2962-2972

4. Kumar SK, Rajkumar SV, Dispenzieri A et al (2008) Improved survival in multiple myeloma and the impact of novel therapies. Blood 111:2516-2520

5. Richardson PG, Sonneveld P, Schuster MW et al (2005) Bortezomib or high-dose dexamethasone for relapsed multiple myeloma. N Engl J Med 352:2487-2498

6. Singhal S, Mehta J, Desikan R et al (1999) Antitumor activity of thalidomide in refractory multiple myeloma. $\mathrm{N}$ Engl $\mathrm{J}$ Med 341:1565-1571

7. Dimopoulos M, Spencer A, Attal M et al (2007) Lenalidomide plus dexamethasone for relapsed or refractory multiple myeloma. N Engl J Med 357:2123-2132

8. Weber DM, Chen C, Niesvizky R et al (2007) Lenalidomide plus dexamethasone for relapsed multiple myeloma in North America. N Engl J Med 357:2133-2142

9. Ocio EM, Mateos MV, Maiso P, Pandiella A, San-Miguel JF (2008) New drugs in multiple myeloma: mechanisms of action and phase I/II clinical findings. Lancet Oncol 9:1157-1165

10. Mitsiades CS, Hideshima T, Chauhan D et al (2009) Emerging treatments for multiple myeloma: beyond immunomodulatory drugs and bortezomib. Semin Hematol 46:166-175

11. Gregoretti IV, Lee YM, Goodson HV (2004) Molecular evolution of the histone deacetylase family: functional implications of phylogenetic analysis. J Mol Biol 338:17-31

12. Dokmanovic M, Clarke C, Marks PA (2007) Histone deacetylase inhibitors: overview and perspectives. Mol Cancer Res 5:981-989

13. de Ruijter AJ, van Gennip AH, Caron HN, Kemp S, van Kuilenburg AB (2003) Histone deacetylases (HDACs): characterization of the classical HDAC family. Biochem J 370:737-749

14. Witt O, Deubzer HE, Milde T, Oehme I (2009) HDAC family: What are the cancer relevant targets? Cancer Lett 277:8-21 
15. Bolden JE, Peart MJ, Johnstone RW (2006) Anticancer activities of histone deacetylase inhibitors. Nat Rev Drug Discov 5:769-784

16. Minucci S, Pelicci PG (2006) Histone deacetylase inhibitors and the promise of epigenetic (and more) treatments for cancer. Nat Rev Cancer 6:38-51

17. Xu WS, Parmigiani RB, Marks PA (2007) Histone deacetylase inhibitors: molecular mechanisms of action. Oncogene 26:5541-5552

18. Hubbert C, Guardiola A, Shao R et al (2002) HDAC6 is a microtubule-associated deacetylase. Nature 417:455-458

19. Tran AD, Marmo TP, Salam AA et al (2007) HDAC6 deacetylation of tubulin modulates dynamics of cellular adhesions. J Cell Sci 120:1469-1479

20. Bali P, Pranpat M, Bradner J et al (2005) Inhibition of histone deacetylase 6 acetylates and disrupts the chaperone function of heat shock protein 90: a novel basis for antileukemia activity of histone deacetylase inhibitors. J Biol Chem 280:26729-26734

21. Esteller M (2008) Epigenetics in cancer. N Engl J Med 358:1148-1159

22. Weichert W, Roske A, Gekeler V et al (2008) Association of patterns of class I histone deacetylase expression with patient prognosis in gastric cancer: a retrospective analysis. Lancet Oncol 9:139-148

23. Weichert W, Roske A, Niesporek S et al (2008) Class I histone deacetylase expression has independent prognostic impact in human colorectal cancer: specific role of class I histone deacetylases in vitro and in vivo. Clin Cancer Res 14:1669-1677

24. Weichert W, Roske A, Gekeler V et al (2008) Histone deacetylases 1,2 and 3 are highly expressed in prostate cancer and HDAC2 expression is associated with shorter PSA relapse time after radical prostatectomy. Br J Cancer 98:604-610

25. Marks P, Rifkind RA, Richon VM, Breslow R, Miller T, Kelly WK (2001) Histone deacetylases and cancer: causes and therapies. Nat Rev Cancer 1:194-202

26. Ozdag H, Teschendorff AE, Ahmed AA et al (2006) Differential expression of selected histone modifier genes in human solid cancers. BMC Genomics 7:90

27. Sakuma T, Uzawa K, Onda T et al (2006) Aberrant expression of histone deacetylase 6 in oral squamous cell carcinoma. Int J Oncol 29:117-124

28. Saji S, Kawakami M, Hayashi S et al (2005) Significance of HDAC6 regulation via estrogen signaling for cell motility and prognosis in estrogen receptor-positive breast cancer. Oncogene 24:4531-4539

29. Zhang Z, Yamashita H, Toyama T et al (2004) HDAC6 expression is correlated with better survival in breast cancer. Clin Cancer Res 10:6962-6968

30. Linggi BE, Brandt SJ, Sun ZW, Hiebert SW (2005) Translating the histone code into leukemia. J Cell Biochem 96:938-950

31. Choi Y, Elagib KE, Goldfarb AN (2005) AML-1-ETO-Mediated erythroid inhibition: new paradigms for differentiation blockade by a leukemic fusion protein. Crit Rev Eukaryot Gene Expr $15: 207-216$

32. Amann JM, Nip J, Strom DK et al (2001) ETO, a target of $\mathrm{t}(8 ; 21)$ in acute leukemia, makes distinct contacts with multiple histone deacetylases and binds $\mathrm{mSin} 3 \mathrm{~A}$ through its oligomerization domain. Mol Cell Biol 21:6470-6483

33. Atsumi A, Tomita A, Kiyoi H, Naoe T (2006) Histone deacetylase 3 (HDAC3) is recruited to target promoters by PML-RARalpha as a component of the N-CoR co-repressor complex to repress transcription in vivo. Biochem Biophys Res Commun 345:1471-1480

34. Chauchereau A, Mathieu M, de Saintignon SJ et al (2004) HDAC4 mediates transcriptional repression by the acute promyelocytic leukaemia-associated protein PLZF. Oncogene 23:8777-8784

35. Xiong W, Wu X, Starnes S et al (2008) An analysis of the clinical and biologic significance of TP53 loss and the identification of potential novel transcriptional targets of TP53 in multiple myeloma. Blood 112:4235-4246
36. Gutierrez NC, Castellanos MV, Martin ML et al (2007) Prognostic and biological implications of genetic abnormalities in multiple myeloma undergoing autologous stem cell transplantation: $\mathrm{t}(4 ; 14)$ is the most relevant adverse prognostic factor, whereas RB deletion as a unique abnormality is not associated with adverse prognosis. Leukemia 21:143-150

37. Chang H, Qi C, Yi QL, Reece D, Stewart AK (2005) p53 gene deletion detected by fluorescence in situ hybridization is an adverse prognostic factor for patients with multiple myeloma following autologous stem cell transplantation. Blood 105:358-360

38. Chng WJ, Price-Troska T, Gonzalez-Paz N et al (2007) Clinical significance of TP53 mutation in myeloma. Leukemia 21:582-584

39. Ocio EM, Maiso P, Chen X et al (2009) Zalypsis: a novel marinederived compound with potent antimyeloma activity that reveals high sensitivity of malignant plasma cells to DNA double-strand breaks. Blood 113:3781-3791

40. Hurt EM, Thomas SB, Peng B, Farrar WL (2006) Reversal of p53 epigenetic silencing in multiple myeloma permits apoptosis by a p53 activator. Cancer Biol Ther 5:1154-1160

41. Davenport EL, Moore HE, Dunlop AS et al (2007) Heat shock protein inhibition is associated with activation of the unfolded protein response pathway in myeloma plasma cells. Blood 110:2641-2649

42. Davenport EL, Morgan GJ, Davies FE (2008) Untangling the unfolded protein response. Cell Cycle 7:865-869

43. Bennett EJ, Bence NF, Jayakumar R, Kopito RR (2005) Global impairment of the ubiquitin-proteasome system by nuclear or cytoplasmic protein aggregates precedes inclusion body formation. Mol Cell 17:351-365

44. Kopito RR (2000) Aggresomes, inclusion bodies and protein aggregation. Trends Cell Biol 10:524-530

45. Kawaguchi Y, Kovacs JJ, McLaurin A, Vance JM, Ito A, Yao TP (2003) The deacetylase HDAC6 regulates aggresome formation and cell viability in response to misfolded protein stress. Cell 115:727-738

46. Miller TA, Witter DJ, Belvedere S (2003) Histone deacetylase inhibitors. J Med Chem 46:5097-5116

47. Rasheed WK, Johnstone RW, Prince HM (2007) Histone deacetylase inhibitors in cancer therapy. Expert Opin Investig Drugs 16:659-678

48. Haggarty SJ, Koeller KM, Wong JC, Grozinger CM, Schreiber SL (2003) Domain-selective small-molecule inhibitor of histone deacetylase 6 (HDAC6)-mediated tubulin deacetylation. Proc Natl Acad Sci USA 100:4389-4394

49. Mitsiades N, Mitsiades CS, Richardson PG et al (2003) Molecular sequelae of histone deacetylase inhibition in human malignant $\mathrm{B}$ cells. Blood 101:4055-4062

50. Mitsiades CS, Mitsiades NS, McMullan CJ et al (2004) Transcriptional signature of histone deacetylase inhibition in multiple myeloma: biological and clinical implications. Proc Natl Acad Sci USA 101:540-545

51. Lavelle D, Chen YH, Hankewych M, DeSimone J (2001) Histone deacetylase inhibitors increase p21(WAF1) and induce apoptosis of human myeloma cell lines independent of decreased IL-6 receptor expression. Am J Hematol 68:170-178

52. Catley L, Weisberg E, Tai YT et al (2003) NVP-LAQ824 is a potent novel histone deacetylase inhibitor with significant activity against multiple myeloma. Blood 102:2615-2622

53. Khan SB, Maududi T, Barton K, Ayers J, Alkan S (2004) Analysis of histone deacetylase inhibitor, depsipeptide (FR901228), effect on multiple myeloma. Br J Haematol 125:156-161

54. Kaiser M, Zavrski I, Sterz J et al (2006) The effects of the histone deacetylase inhibitor valproic acid on cell cycle, growth suppression and apoptosis in multiple myeloma. Haematologica 91:248-251

55. Schwartz C, Palissot V, Aouali N et al (2007) Valproic acid induces non-apoptotic cell death mechanisms in multiple myeloma cell lines. Int J Oncol 30:573-582 
56. Kitazoe K, Abe M, Hiasa M et al (2009) Valproic acid exerts antitumor as well as anti-angiogenic effects on myeloma. Int $\mathbf{J}$ Hematol 89:45-57

57. Neri P, Tagliaferri P, Di Martino MT et al (2008) In vivo antimyeloma activity and modulation of gene expression profile induced by valproic acid, a histone deacetylase inhibitor. $\mathrm{Br} \mathrm{J}$ Haematol 143:520-531

58. Catley L, Weisberg E, Kiziltepe T et al (2006) Aggresome induction by proteasome inhibitor bortezomib and alpha-tubulin hyperacetylation by tubulin deacetylase (TDAC) inhibitor LBH589 are synergistic in myeloma cells. Blood 108:3441-3449

59. Maiso P, Carvajal-Vergara X, Ocio EM et al (2006) The histone deacetylase inhibitor LBH589 is a potent antimyeloma agent that overcomes drug resistance. Cancer Res 66:5781-5789

60. Ocio EM, Vilanova D, Atadja P et al (2009) In vitro and in vivo rationale for the triple combination of panobinostat (LBH589) and dexamethasone with either bortezomib or lenalidomide in multiple myeloma. Haematologica

61. Feng R, Oton A, Mapara MY, Anderson G, Belani C, Lentzsch S (2007) The histone deacetylase inhibitor, PXD101, potentiates bortezomib-induced anti-multiple myeloma effect by induction of oxidative stress and DNA damage. Br J Haematol 139:385-397

62. Arts J, Angibaud P, Marien A et al (2007) R306465 is a novel potent inhibitor of class I histone deacetylases with broadspectrum antitumoral activity against solid and haematological malignancies. Br J Cancer 97:1344-1353

63. Feng R, Ma H, Hassig CA et al (2008) KD5170, a novel mercaptoketone-based histone deacetylase inhibitor, exerts antimyeloma effects by DNA damage and mitochondrial signaling. Mol Cancer Ther 7:1494-1505

64. Deleu S, Lemaire M, Arts J et al (2009) The effects of JNJ26481585 , a novel hydroxamate-based histone deacetylase inhibitor, on the development of multiple myeloma in the 5T2MM and 5T33MM murine models. Leukemia 23:1894-1903

65. Todoerti K, Barbui V, Pedrini $\mathrm{O}$ et al Pleiotropic anti-myeloma activity of ITF2357: inhibition of interleukin-6 receptor signaling and repression of miR-19a and miR-19b. Haematologica 95:260-269

66. Mandl-Weber S, Meinel F, Jankowsky R, Oduncu F, Schmidmaier $\mathrm{R}$, Baumann P The novel inhibitor of histone deacetylase resminostat (RAS2410) inhibits proliferation and induces apoptosis in multiple myeloma (MM) cells. Br J Haematol

67. Fandy TE, Shankar S, Ross DD, Sausville E, Srivastava RK (2005) Interactive effects of HDAC inhibitors and TRAIL on apoptosis are associated with changes in mitochondrial functions and expressions of cell cycle regulatory genes in multiple myeloma. Neoplasia 7:646-657

68. Akiyama M, Hideshima T, Hayashi T et al (2002) Cytokines modulate telomerase activity in a human multiple myeloma cell line. Cancer Res 62:3876-3882

69. Chen S, Dai Y, Pei XY, Grant S (2009) Bim upregulation by histone deacetylase inhibitors mediates interactions with the Bcl-2 antagonist ABT-737: evidence for distinct roles for Bcl-2, Bcl-xL, and Mcl-1. Mol Cell Biol 29:6149-6169

70. Damiano JS, Cress AE, Hazlehurst LA, Shtil AA, Dalton WS (1999) Cell adhesion mediated drug resistance (CAM-DR): role of integrins and resistance to apoptosis in human myeloma cell lines. Blood 93:1658-1667

71. Hideshima T, Mitsiades C, Tonon G, Richardson PG, Anderson KC (2007) Understanding multiple myeloma pathogenesis in the bone marrow to identify new therapeutic targets. Nat Rev Cancer 7:585-598

72. Wolf JL, Siegel D, Matous J et al (2008) A Phase II Study of Oral Panobinostat (LBH589) in Adult Patients with Advanced Refractory Multiple Myeloma. ASH Annu Meet Abstr 112:2774
73. Richardson P, Mitsiades C, Colson K et al (2008) Phase I trial of oral vorinostat (suberoylanilide hydroxamic acid, SAHA) in patients with advanced multiple myeloma. Leuk Lymphoma 49:502-507

74. Galli M, Salmoiraghi S, Golay J et al A phase II multiple dose clinical trial of histone deacetylase inhibitor ITF2357 in patients with relapsed or progressive multiple myeloma. Ann Hematol 89:185-190

75. Niesvizky R, Ely S, DiLiberto M et al (2005) Multicenter Phase II Trial of the Histone Deacetylase Inhibitor Depsipeptide (FK228) for the Treatment of Relapsed or Refractory Multiple Myeloma (MM). ASH Annu Meet Abstr 106:2574-

76. Campbell RA, Sanchez E, Steinberg J et al Vorinostat enhances the antimyeloma effects of melphalan and bortezomib. Eur J Haematol 84:201-211

77. Deleu S, Lemaire M, Arts J et al (2009) Bortezomib alone or in combination with the histone deacetylase inhibitor JNJ-26481585: effect on myeloma bone disease in the 5T2MM murine model of myeloma. Cancer Res 69:5307-5311

78. Hideshima T, Bradner JE, Wong J et al (2005) Small-molecule inhibition of proteasome and aggresome function induces synergistic antitumor activity in multiple myeloma. Proc Natl Acad Sci USA 102:8567-8572

79. Pei XY, Dai Y, Grant S (2004) Synergistic induction of oxidative injury and apoptosis in human multiple myeloma cells by the proteasome inhibitor bortezomib and histone deacetylase inhibitors. Clin Cancer Res 10:3839-3852

80. Badros A, Burger AM, Philip S et al (2009) Phase I study of vorinostat in combination with bortezomib for relapsed and refractory multiple myeloma. Clin Cancer Res 15:5250-5257

81. Weber D, Badros AZ, Jagannath S et al (2008) Vorinostat plus bortezomib for the treatment of relapsed/refractory multiple myeloma: early clinical experience. ASH Annu Meet Abstr 112:871

82. Weber DM, Jagannath S, Sobecks R et al (2008) Combination of vorinostat plus bortezomib for the treatment of patients with multiple myeloma who have previously received bortezomib. ASH Annu Meet Abstr 112:3711

83. San-Miguel JF, Sezer O, Siegel D et al (2009) A phase IB, multicenter, open-label dose-escalation study of oral panobinostat (LBH589) and I.V. Bortezomib in patients with relapsed multiple myeloma. ASH Annu Meet Abstr 114:3852-

84. Harrison SJ, Quach H, Yuen K et al (2008) High response rates with the combination of bortezomib, dexamethasone and the panhistone deacetylase inhibitor romidepsin in patients with relapsed or refractory multiple myeloma in a phase I/II clinical trial. ASH Annu Meet Abstr 112:3698

85. Voorhees PM, Gasparetto C, Richards KL et al (2009) Vorinostat in combination with pegylated liposomal doxorubicin and bortezomib for patients with relapsed/refractory multiple myeloma: results of a phase I study. ASH Annu Meet Abstr 114:306-

86. Spencer A, Lonial S, Taylor K et al (2009) Panobinostat and lenalidomide combination phase I trial in myeloma. Clin Lymphoma Myeloma. Abstract 329

87. Siegel D, Weber DM, Mitsiades CS et al (2009) Combined vorinostat, lenalidomide and dexamethasone therapy in patients with relapsed or refractory multiple myeloma: a phase I study. ASH Annu Meet Abstr 114:305-

88. Berenson JR, Yellin O, Boccia RV, Nassir Y, Rothstein S, Swift RA (2009) A phase I study of oral melphalan combined with LBH589 for patients with relapsed or refractory multiple myeloma (MM). ASH Annu Meet Abstr 114:1855- 